\title{
Quantum Quench and Prethermalization Dynamics in a Two-Dimensional Fermi Gas with Long-Range Interactions
}

\author{
N. Nessi, ${ }^{1}$ A. Iucci, ${ }^{1}$ and M. A. Cazalilla, ${ }^{2, *}$ \\ ${ }^{1}$ Instituto de Física La Plata (IFLP)-CONICET and Departamento de Física, Universidad Nacional de La Plata, \\ CC 67, 1900 La Plata, Argentina \\ ${ }^{2}$ Department of Physics, National Tsing Hua University, and National Center for Theoretical Sciences (NCTS), \\ Hsinchu City, Taiwan \\ (Received 18 January 2014; published 17 November 2014)
}

\begin{abstract}
We study the effect of suddenly turning on a long-range interaction in a spinless Fermi gas in two dimensions. The short- to intermediate-time dynamics is described using the method of bosonization of the Fermi surface. The space-time dependence of the nonequilibrium fermion density matrix as well as the evolution after the quench of the discontinuity at the Fermi momentum of the momentum distribution are computed. We find that the asymptotic state predicted by bosonization is consistent with the existence of a prethermalization plateau, which is also predicted by a perturbative approach in terms of the fermionic degrees of freedom. The bosonized representation, however, explicitly allows for the construction of the generalized Gibbs ensemble describing the prethermalized state.
\end{abstract}

Prethermalization [1] is a fast loss of memory of the initial conditions due to dephasing after a system has been driven out of equilibrium. Recently, it has been associated with the emergence of quasistationary states that precede the true thermalization [2-10]. The understanding of how such a state emerges is relevant to fields as diverse as heavy ion collisions [1,11] and nonequilibrium phenomena in condensed matter and atomic systems [12,13]. Currently, the subject is also attracting a great deal of attention in connection with the thermalization time scales of ultracold atomic systems undergoing a quantum quench [14-17]. These systems are ideal for the study of dynamics of isolated many-body systems as they are decoupled from their environment and can remain quantum coherent for rather long times [13]. Indeed, prethermalization has been recently observed in an experiment probing the nonequilibrium dynamics of a one-dimensional quantum Bose gas [16,17]. Theoretically, it was also reported in early studies of interaction quantum quenches in the Fermi Hubbard model [2-4].

The existence of prethermalization implies that the complete thermalization of a system driven out of equilibrium takes place in (at least) two stages. The first stage is dominated by the inertial response of the system followed by rapid dephasing. The second stage is dominated by inelastic particle collisions, which break the conservation laws that temporarily hold during the first stage. The collisions are therefore responsible for driving the system to the thermal state. As pointed out early on by Kehrein and Moeckel [2,3] and recently discussed by others [18], the crossover from the prethermalized to the fully thermalized state can be described using a kinetic equation approach.

As far as the prethermalized state is concerned, several groups have suggested $[10,17,19]$ that it can be described in terms of a generalized Gibbs ensemble (GGE). The latter applies in the long-time regime following a quantum quench in integrable models [20-24]. However, the connection between the GGE and prethermalization is still rather poorly understood. For instance, in the theoretical studies of Refs. [2-4], the set of integrals of motion required for the construction of the GGE has not been identified. Furthermore, most of the available results are either for one- or infinite-dimensional systems $[2-4,25]$ and results in dimensions $d=2,3$ are scarce despite that these dimensions are the most interesting from the experimental point of view.

In this work, we undertake the study of an interaction quench in a two-dimensional Fermi liquid with long-range interactions. As we show below, during the first stage leading to the prethermalized state, the system dynamics can be approximated by an integrable model, even when the Hamiltonian is not integrable and ultimately should thermalize at long times [26]. An important by-product is the set of integrals of motion that allow the description of the prethermalized state in terms of the GGE. The GGE-based description provides an economical way of encoding the quantum correlations that build up during the initial stages of the evolution of the system. Equally importantly, it also explains how prethermalization proceeds from the initial state by relating it to an exactly solvable version of the system. The construction of the GGE is carried out using the Fermi surface (FS) bosonization method [27-33], which is applied to an exactly solvable truncation of the Hamiltonian describing the dynamics. Because the time evolution after suddenly switching on a long-range interaction creates a very large number of low-energy and momentum particle-hole pairs, the FS bosonization 
provides an accurate description of the short-time dynamics leading to prethermalization. Experimentally, our results can be relevant for realistic systems such as dipolar quantum gases of fermionic atoms [34,35]. The discussion of the experimental consequences will be given elsewhere.

The dynamics of the system at all times $t>0$ is dictated by the Hamiltonian $H=H_{0}+H_{\text {int }}$ that describes a gas of spinless fermions interacting via a two-body potential with

$$
\begin{aligned}
H_{0} & =\sum_{\boldsymbol{k}} \epsilon(\boldsymbol{k}) c_{\boldsymbol{k}}^{\dagger} c_{\boldsymbol{k}}, \\
H_{\mathrm{int}} & =\frac{1}{V} \sum_{\boldsymbol{k}_{1}, \boldsymbol{k}_{2}, \boldsymbol{q}} f(q) Q_{\boldsymbol{k}_{1}}(\boldsymbol{q}) Q_{\boldsymbol{k}_{2}}(-\boldsymbol{q}),
\end{aligned}
$$

where $c_{k}, c_{k}^{\dagger}$ are the fermionic field operators, $\epsilon(\boldsymbol{k})$ is the dispersion relation, $f(q)$ is the (isotropic) two-body interaction that is switched on at $t=0$ and $Q_{\boldsymbol{k}}(\boldsymbol{q})=c_{\boldsymbol{k}+\boldsymbol{q}}^{\dagger} c_{\boldsymbol{k}}$ create particle-hole excitations. The initial condition is the ground state $\left|\Psi_{0}\right\rangle$ of $H_{0}$, i.e., the filled Fermi sea with all states occupied up to momentum $k_{F}=\sqrt{4 \pi \rho_{0}}$, where $\rho_{0}=$ $\mathcal{N} / V$ is the mean fermion density. We assume that the interaction is weak and long-ranged: $f(q)=f_{0} F(q)$, where $f_{0}$ is small compared to the Fermi energy and $F(q)$ decays rapidly for $q \gg q_{c}$, where $q_{c}^{-1} \gg k_{F}^{-1}$ is the spatial range of the interaction.

For a weak interaction quench, we first compute the time evolution of specific observables using a perturbative approach in the fermionic degrees of freedom similar to the one used in Refs. [2,3], where it was applied to the Hubbard model. Below, we show that the results from the perturbative approach and FS bosonization are fully consistent with each other. To leading order in $f_{0}$, we obtain the following result for the discontinuity of the momentum distribution at $k=k_{F}$ [36]:

$$
Z^{\mathrm{neq}}(t)=1-4 \int_{-\infty}^{\infty} d E \frac{\sin ^{2}(E t / 2)}{E^{2}} \Gamma_{\boldsymbol{k}_{F}}(E)+\mathcal{O}\left(f_{0}^{3}\right),
$$

where $\Gamma_{\boldsymbol{k}_{F}}(E)$ can be related to the imaginary part of the self-energy of a particle (hole) with energy $E>0(E<0)$ and momentum $\boldsymbol{k}$ on the Fermi surface, as shown in the Supplemental Material [36].

Let us first consider the short-and long-time behaviors of Eq. (2). For short times, $Z^{\mathrm{eq}}(t)=1-c t^{2}$, where $c \propto f_{0}^{2}$. However, the long-time behavior is determined by the behavior of $\Gamma_{\boldsymbol{k}_{F}}(E)$ as $E \rightarrow 0$. According to Fermi-liquid theory [37], $\Gamma_{\boldsymbol{k}_{F}}(E \rightarrow 0) \sim E^{2+b}$ with $b=0$. It is instructive, however, to consider the behavior of $Z^{\text {neq }}(t)$ for arbitrary $b$. Thus, for $b>-1$ we will get a finite value of $Z^{\text {neq }}(\infty)$ while for $b<-1$ the time-dependent term in Eq. (2) diverges for large $t$; i.e., it gives rise to a secular term in the perturbative expansion. For a circular FS in two dimensions and an interaction $F(q) \sim e^{-q / q_{c}}, b=0$ and $Z^{\text {neq }}(t)=Z^{\text {st }}=$ const as $t \rightarrow+\infty$. Moreover, the calculation shows that the nonequilibrium steady state and the ground state values are related by means of $1-Z^{\text {st }}=2\left(1-Z^{\text {eq }}\right)$ [36]. The constant $Z^{\text {st }}$ is approached, for large $t$ as $Z^{\text {neq }}(t)-Z^{\text {st }} \sim f_{0}^{2} / t$, which follows from $\Gamma_{\boldsymbol{k}_{F}}(E \rightarrow 0) \sim E^{2}$. The behavior just described suggests the existence a plateau in $Z^{\text {neq }}(t)$, which has been interpreted in earlier work [2-4] as an indication of the prethermalized state.

Next, we present a description of the dynamics based on FS bosonization. This method allows for a rederivation of the above results while going beyond leading order in $f_{0}$ through an effective resummation of a certain perturbative subseries. Furthermore, it also allows us to obtain the effective integrable Hamiltonian that describes the dynamics in the prethermalized regime and the associated conserved quantities. To set up the stage, consider an interparticle collision with momentum transfer $\lambda$. The rate of occurrence of such scattering events is $\sim f_{0}^{2}|F(\lambda)|^{2} N(0) \sim \tau^{-1}$, where $N(0)=\left(k_{F} / 2 \pi v_{F}\right)$ is the density of states at the Fermi energy. For $\lambda>q_{c}$ such processes are strongly suppressed by the long-range nature of the interaction. Therefore, their influence on the dynamics is negligible for $t \lesssim \tau$. This observation implies that (i) given the initial condition, at short times we can neglect scattering processes that transfer fermions outside a shell of thickness $\lambda>q_{c}$ around the FS and (ii) inside this shell only forward scattering processes are relevant. In order to clarify point (ii) we further divide the shell into $N$ patches of dimensions $\Lambda \gg \lambda$ along the FS that are labeled by a vector $S$. The scattering processes contained in $H_{\text {int }}$ with momentum transfer $q<\lambda$ are thus of two types: forward scattering (fermions remain in the same patch) and exchange scattering between neighboring patches. Both types of processes conserve the number of fermions in each patch, which in the limit of a large number of patches becomes a $U(1)_{k}^{N}$ symmetry [27-29]. However, given the squat aspect ratio of the patches $(\Lambda \gg \lambda)$ the exchange scattering between neighboring patches can be also neglected since they amount to a small correction to the forward scattering processes that occur in the bulk of the patches [36].

In addition, the above FS sectorization allows us to linearize the dispersion relation within each patch, provided that the patch size $\Lambda$ is small enough compared to the scale in which the FS changes its shape (in our case it is enough to set $\Lambda \ll k_{F}$ ). The truncated Hamiltonian can be written in the form [27-29]:

$$
H=\sum_{S, \boldsymbol{T}, \boldsymbol{q}} J_{S}(\boldsymbol{q})\left(\frac{v_{F}}{2 \Omega} \delta_{S, \boldsymbol{T}}+\frac{f(q)}{V}\right) J_{\boldsymbol{T}}(-\boldsymbol{q}),
$$

where $v_{F}=\left|\nabla_{\boldsymbol{k}} \epsilon(\boldsymbol{k})\right|_{|\boldsymbol{k}|=k_{F}}$ is the Fermi velocity and $J_{\boldsymbol{S}}(\boldsymbol{q})$ are the Fourier components of the density operator corresponding to momenta within the patch $S$ [36]. Thus, these operators create particle-hole pairs near the FS with 
momentum $q$ within a distance $\Lambda$ of the direction $S, \Omega=$ $\Lambda\left(V /(2 \pi)^{2}\right)$ ( $V$ is the area of the system). Notice that the collection of conditions over the cutoffs is consistent and reads $k_{F} \gg \Lambda \gg \lambda>q_{c}$. We therefore expect that the approximations described above will be accurate for high densities (large $k_{F}$ ).

The truncation of the Hamiltonian described above is similar to the one carried out in Fermi-liquid theory (FLT) [27-29,37]. However, an important difference is that our truncated Hamiltonian is written in terms of the bare interaction and the bare fermionic degrees of freedom, instead of a renormalized interaction between Landau quasiparticles. The resulting Hamiltonian is therefore different from the Hamiltonian that describes the low energy degrees of freedom close to the ground state of (1). It is also worth noting that this argument not only applies to $d=2$, but also to $d=1$ where, interestingly, it has been recently found that the dynamics following an interaction quench is described by the bosonic low-energy field theory parametrized with the bare parameters of the microscopic Hamiltonian instead of the renormalized equilibrium parameters [37].

The usefulness of the FS bosonization stems from the observation that the fermionic currents $J_{S}(\boldsymbol{q})$ obey a set of anomalous commutation relations, which become a bosonic algebra for $\Lambda \gg \lambda$ [27-32]. This allows for a representation of the currents in terms of Tomonaga bosons, which renders the Hamiltonian in (2) quadratic. Higher order (i.e., anaharmonic) corrections to (3) such as those arising from the curvature of the FS and the fermion dispersion, introduce interactions between the Tomonaga bosons [33]. These terms break the set of conservation laws that make the above Hamiltonian exactly solvable [27-29]. As a consequence, a complete relaxation to thermal equilibrium is thus expected to occur at long times. The latter phenomenon is controlled only by the conservation laws that are respected by the true fermion Hamiltonian, which differs from the truncated integrable Hamiltonian because it also describes inelastic collisions between the fermions.

In order to calculate correlation functions, we must solve the Heisenberg equations of motion for the bosonic operators. This is equivalent to diagonalizing the bilinear Hamiltonian (3), which is is not analytically possible because all patches are coupled by the interaction. The explicit diagonalization can be bypassed by noticing that the solution to the equations of motion can be written exclusively in terms of the equilibrium retarded correlation functions between the bosonic fields, which follows from the bilinear form of the Hamiltonian [36]. Those retarded correlations can be calculated exactly using the imaginary time path integral formulation of the bosonic problem [27-29].

We first discuss the asymptotic state of the system at long times (formally, for $t \rightarrow+\infty$ ), and argue that its properties are consistent with those of a prethermalized state. To this end we consider the in-patch nonequilibrium density matrix
$\mathcal{G}_{\boldsymbol{S}}^{\text {neq }}(\boldsymbol{x}, t)=\left\langle\Psi_{0}\left|e^{i H t} \psi_{\boldsymbol{S}}^{\dagger}(\boldsymbol{x}) \psi_{\boldsymbol{S}}(\mathbf{0}) e^{i H t}\right| \Psi_{0}\right\rangle$, where $\psi_{\boldsymbol{S}}(\boldsymbol{x})$ is the fermion field operator that annihilates a fermion at position $\boldsymbol{x}$ with momenta within the patch $\boldsymbol{S}$. The correlation function of the physical fermions can be obtained summing over all patches [27-29]. The calculation can be carried out by means of the bosonic representation of the fermionic field [27-32]. We find that $\mathcal{G}_{S}^{\text {neq }}(\boldsymbol{x}, t)=$ $\mathcal{G}_{S}^{0}(\boldsymbol{x}) Z_{S}^{\text {neq }}(\boldsymbol{x}, t)$, where $\mathcal{G}_{S}^{0}(\boldsymbol{x})$ is the in-patch free correlator (i.e., the initial condition) and

$$
\begin{aligned}
Z_{S}^{\mathrm{neq}}(\boldsymbol{x}, t)= & \exp \left[\frac{1}{\Omega} \sum_{\boldsymbol{q}, \hat{\boldsymbol{n}}_{S} \cdot \boldsymbol{q}>0, \boldsymbol{T}} \frac{\left|\tilde{G}_{\boldsymbol{S} \boldsymbol{T}}^{\mathrm{ret}, \mathrm{eq}}(\boldsymbol{q}, t)\right|^{2}}{\hat{\boldsymbol{n}}_{\boldsymbol{S}} \cdot \boldsymbol{q}}\right. \\
& \times 2(\cos (\boldsymbol{q} \cdot \boldsymbol{x})-1)],
\end{aligned}
$$

where $\tilde{G}_{\boldsymbol{S T}}^{\text {ret,eq }}(\boldsymbol{q}, t)$ is the (equilibrium) anomalous retarded correlation function of the Tomonaga bosons and $\hat{\boldsymbol{n}}_{S}$ is the unit normal to the FS at patch $\boldsymbol{S}$. Although it is possible to obtain an expression for $\tilde{G}^{\text {ret,eq }}$ valid at all orders in $f_{0}$, in order to render the expressions analytically tractable and make contact with the perturbative results described above, we work only at the leading order in $f_{0}$. Thus,

$$
\begin{aligned}
\left|\tilde{G}_{\boldsymbol{S T}}^{\mathrm{ret}, \mathrm{eq}}(\boldsymbol{q}, t)\right|^{2}= & \frac{2 f_{0}^{2} F(q)^{2} \Lambda^{2}}{(2 \pi)^{4} v_{F}^{2}} \mathcal{P} \mathcal{V} \frac{\left|\hat{\boldsymbol{n}}_{\boldsymbol{S}} \cdot \boldsymbol{q}\right|\left|\hat{\boldsymbol{n}}_{\boldsymbol{T}} \cdot \boldsymbol{q}\right|}{\left[\left(\hat{\boldsymbol{n}}_{S}-\hat{\boldsymbol{n}}_{\boldsymbol{T}}\right) \cdot \boldsymbol{q}\right]^{2}} \\
& \times \sin ^{2}\left[\left(\hat{\boldsymbol{n}}_{\boldsymbol{S}}-\hat{\boldsymbol{n}}_{\boldsymbol{T}}\right) \cdot \boldsymbol{q} \frac{v_{F} t}{2}\right]+\mathcal{O}\left(f_{0}^{3}\right),
\end{aligned}
$$

where $\mathcal{P V}$ indicates that in the limit of large number of patches, where patch sums can be approximated by angular integrals, we must take the principal value [30].

Next, we relate the stationary state and the ground state correlations. Thus, considering the correlation function $\mathcal{G}^{\mathrm{eq}}(\boldsymbol{x})=\left\langle\Psi\left|\psi^{\dagger}(\boldsymbol{x}) \psi(\mathbf{0})\right| \Psi\right\rangle \quad(|\Psi\rangle$ is the ground state of $H$ ), we define $Z^{\text {eq }}(x)=\mathcal{G}^{\text {eq }}(x) / \mathcal{G}^{0}(x)$. Furthermore, due to dephasing, the oscillatory part of $\left|\tilde{G}_{S T}^{\text {ret,eq }}(\boldsymbol{q}, t)\right|^{2}$ in Eq. (4) drops out for $t \rightarrow \infty$ [36]. Thus, we find a simple relation between the stationary nonequilibrium and the ground state correlations

$$
\lim _{t \rightarrow \infty} \ln \left[Z^{\text {neq }}(x, t)\right]=2 \ln \left[Z^{\mathrm{eq}}(x)\right]+\mathcal{O}\left(f_{0}^{3}\right),
$$

where $Z^{\text {neq }}(x, t)=\mathcal{G}^{\text {neq }}(x, t) / \mathcal{G}^{0}(x)$. Note that, owing to rotation invariance, both $Z^{\text {eq }}$ and $Z^{\text {neq }}$ depend only on $x=|\boldsymbol{x}|$. Taking $x \rightarrow \infty$, Eq. (6) leads to a relation between the discontinuity at $k_{F}$ in the momentum distribution of the stationary and ground states: $Z^{\text {neq }} \simeq\left(Z^{\text {eq }}\right)^{2}$. Recalling that $Z^{\mathrm{eq}}=1-\mathcal{O}\left(f_{0}^{2}\right)$, we retrieve the perturbative result discussed above.

Exponentiating Eq. (6) and further developing in powers of the interaction strength, it is possible to obtain a simple relation between the stationary state $\left[n^{\text {st }}(k)\right]$ and ground 
state $\left[n^{\mathrm{eq}}(k)\right]$ momentum distributions, $2\left[n^{\mathrm{eq}}(k)-n_{0}^{\mathrm{eq}}(k)\right]=$ $n^{\mathrm{st}}(k)-n_{0}^{\mathrm{eq}}(k)+\mathcal{O}\left(f_{0}^{3}\right)$, where $n_{0}^{\mathrm{eq}}(k)=\theta\left(k_{F}-k\right)$. A similar result was obtained in Refs. [2,3] for the Hubbard model under very different assumptions (i.e., a short-range interaction between spinful fermions). In particular, this relation implies that, to lowest order in the interaction strength, all the energy injected into the system by the quench, $E_{\text {ex }}=E_{\text {neq }}-E_{\text {eq }}$, where $E_{\text {neq }}=\left\langle\Psi_{0}|H| \Psi_{0}\right\rangle=0$ and $E_{\mathrm{eq}}=\langle\Psi|H| \Psi\rangle$, is transformed into kinetic energy in the stationary (prethermalized) state [2,3].

Let us next discuss the description of the prethermalized state using the GGE. In our framework, the GGE description of the prethermalized state arises naturally since the truncated Hamiltonian is a (bosonic) bilinear and, consequently, dephasing implies that all correlations in the steady state are described by a GGE [24,38]. If we denote with $\left\{\alpha_{l}(\boldsymbol{q}), \alpha_{l}^{\dagger}(\boldsymbol{q})\right\}$ the bosonic basis that diagonalizes the Hamiltonian in Eq. (3), the GGE density matrix can be written as

$$
\rho_{\mathrm{GGE}}=\frac{1}{Z_{\mathrm{GGE}}} \exp \left[\sum_{l, \boldsymbol{q}} \lambda_{l}(\boldsymbol{q}) I_{l}(\boldsymbol{q})\right]
$$

where $I_{l}(\boldsymbol{q})=\alpha_{l}^{\dagger}(\boldsymbol{q}) \alpha_{l}(\boldsymbol{q})$ are the conserved quantities, $Z_{\mathrm{GGE}}=\operatorname{Tr}\left[\rho_{\mathrm{GGE}}\right]$ and the Lagrange multipliers $\lambda_{l}(\boldsymbol{q})$ are obtained from the initial conditions, $\left\langle I_{l}(\boldsymbol{q})\right\rangle_{t=0}=$ $\left\langle\Psi_{0}\left|I_{l}(\boldsymbol{q})\right| \Psi_{0}\right\rangle=\operatorname{Tr}\left[\rho_{\mathrm{GGE}} I_{l}(\boldsymbol{q})\right]$. We also have explicitly checked that the density matrix (7) reproduces all the studied quantities in the prethermalized state. It is also worth noting that the conserved quantities can be refermionized, at least formally. Using the matrix transformation that diagonalizes the Hamiltonian, they can be expressed as a linear combination of products of two-patch densities.

The close relationship linking prethermalization, dephasing dynamics, and GGE for a generic Fermi liquid of finite dimensionality unveiled within our approach is very similar to the phenomena observed in exactly solvable models in one space dimension (1D) [24,38]. Indeed, for 1D systems recent numerical studies [37,39] have shown that the bosonization description provided in Refs. [21-23] is fairly accurate and universal, and that even the dynamics at intermediate times can be described by the GGE. For longer times, mode coupling has been argued to lead to thermalization [40].

It is well known that after a sudden quench in 1D systems the correlations propagate at a finite speed giving rise to the so-called "light-cone effect" [41]. In higher dimensions, this phenomenon is largely unexplored [42]. Starting from Eqs. (4) and (5) it can be shown [36] that in the spatiotemporal region defined by $x \gg 2 v_{F} t$ we can approximately neglect the spatial dependence of $Z_{\boldsymbol{S}}^{\text {neq }}(\boldsymbol{x}, t)$ and, with it, the patch index. Outside the light cone, $x \gg 2 v_{F} t$, the interaction correction is therefore approximately the same for all patches: $Z_{S}^{\text {neq }}(x, t) \approx Z^{\text {neq }}(t)$ and the full correlation function thus reads $\mathcal{G}^{\text {neq }}(x, t) \approx$ $\mathcal{G}^{0}(x) Z^{\text {neq }}(t)$; i.e., the correlations retain the same spatial dependence as in the initial state up to a time-dependent prefactor. This factor defines the time-dependent quasiparticle residue that is analyzed below. In the opposite limit, $x \ll 2 v_{F} t$, we can neglect the temporal dependence and the steady state correlations dominate: $Z^{\text {neq }}(x, t) \approx$ $\lim _{t \rightarrow \infty} Z^{\text {neq }}(x, t)$. We conclude that, at short times, the correlations will travel isotropically with the bare Fermi velocity of the fermions.

Finally, we consider the dynamics of the discontinuity of the momentum distribution at $k=k_{F}$. For short times $v_{F} t \ll q_{c}^{-1}$ we find a Gaussian decay of $Z^{\text {neq }}(t)$ from its initial value of one:

$Z^{\text {neq }}(t)=\exp \left[-t^{2} \frac{4 f_{0}^{2} k_{F}}{(2 \pi)^{4}} c \int_{0}^{\infty} d q(F(q) q)^{2}+\mathcal{O}\left(f_{0}^{3}\right)\right]$,

where $c$ is an $\mathcal{O}(1)$ constant that stems from the angular integration over the FS. The Gaussian decay at short times is independent of the form of the interaction and it also occurs in one dimension [21-23]. For $v_{F} t \gg q_{c}^{-1}$, the explicit form of the interaction is required. For a simple form, $f(q)=f_{0}\left(q / q_{c}\right)^{n} e^{-q / q_{c}}$, we find

$$
Z^{\text {neq }}(t) \approx Z^{\text {st }} \exp \left[g^{2} a_{n}\left(v_{F} q_{c} t\right)^{-(2 n+1)}\right],
$$

where $Z^{\text {st }} \simeq\left(Z^{\mathrm{eq}}\right)^{2}$ is the stationary-state quasiparticle residue, $a_{n}$ a positive constant coming from the angular integral and we have defined the dimensionless coupling constant $g=\left(f_{0} \sqrt{k_{F} q_{c}} / 2 \pi^{2} v_{F}\right)$. We note that expanding in powers of $f_{0}$ Eqs. (8) and (9), we recover the same asymptotic behavior for $Z^{\text {neq }}(t)$ as obtained using perturbation theory. In Fig. 1 we illustrate the dynamics of the quasiparticle residue for different interaction strengths and $n=0$.

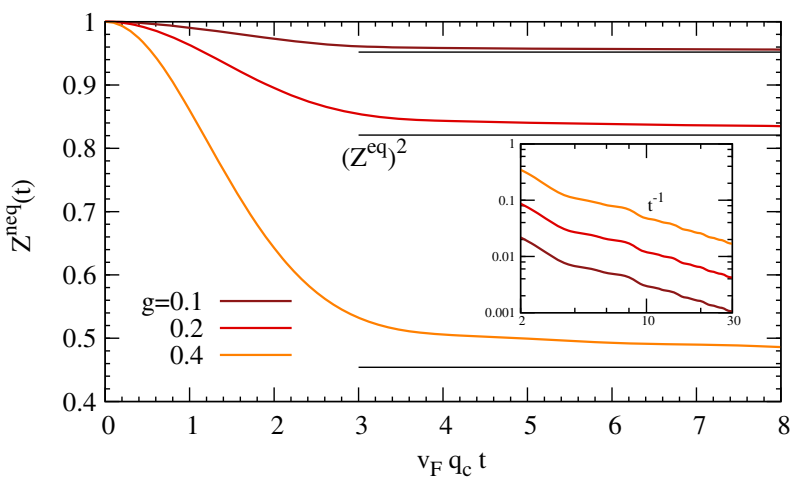

FIG. 1 (color online). Discontinuity of the zero-temperature momentum distribution at the Fermi momentum, $Z^{\text {neq }}(t)$, as a function of time $t$ for several values of $g=f_{0} \sqrt{k_{F} q_{c}} /\left(2 \pi^{2} v_{F}\right)$. $Z^{\text {neq }}(t)$ exhibits a Gaussian decay at short times. Asymptotically, it saturates to a finite value $Z^{\text {st }} \simeq\left(Z^{\text {eq }}\right)^{2}$ (horizontal lines) in the stationary (prethermalized) state. Inset: $\ln \left[Z^{\text {neq }}(t) / Z^{\text {st }}\right]$ showing that $\ln Z^{\text {neq }}(t) \sim t^{-1}$ asymptotically. 
The picture that emerges from the bosonization treatment of the two-dimensional Fermi liquid suggests that the first stage following the quench can be regarded as a collective nonequilibrium quasiparticle dressing by the interactions of the noninteracting fermions (or, for that matter, the bare quasiparticles) that are the fermionic excitations of the initial Hamiltonian. A similar behavior of $Z^{\text {neq }}(t)$ has been observed in interaction quenches in one dimension [21-23,39], but a major difference with the present case is that $Z_{1 D}^{\text {neq }}(t \rightarrow+\infty)=Z^{\text {st }}=0$, indicating the nonequilibrium dressing leads to a complete destruction of the fermionic quasiparticles in the system.

In conclusion, we have studied the quench dynamics of a Fermi gas with long-range interactions using Fermi surface (FS) bosonization. We were able to obtain the full spacetime dependence of the nonequilibrium density matrix as well as the evolution of (the zero-temperature) discontinuity of the momentum distribution at $k=k_{F}$ after the quench. We have shown that prethermalization can be understood as the result of dephasing between the bosonic FS excitations. Furthermore, the statistical description of the prethermalized state in terms of the generalized Gibbs ensemble has been obtained and the integrals of motion needed for its construction have been identified as the eigenmodes of the bosonic FS Hamiltonian.

This work was partially supported by CONICET (PIP 0662), ANPCyT (PICT 2010-1907), and UNLP (PID X497), Argentina. M. A.C. acknowledges financial support from National Science Council of Taiwan, the National Center for Theoretical Sciences (NCTS) of Taiwan, and a start-up fund from the National Tsing Hua University.

*Corresponding author. miguel.cazalilla@gmail.com

[1] J. Berges, S. Borsányi, and C. Wetterich, Phys. Rev. Lett. 93, 142002 (2004).

[2] M. Moeckel and S. Kehrein, Phys. Rev. Lett. 100, 175702 (2008).

[3] M. Moeckel and S. Kehrein, Ann. Phys. (Amsterdam) 324, 2146 (2009).

[4] M. Eckstein, M. Kollar, and P. Werner, Phys. Rev. Lett. 103, 056403 (2009).

[5] J. Lux, J. Muller, A. Mitra, and A. Rosch, Phys. Rev. A 89, 053608 (2014).

[6] J. Marino and A. Silva, Phys. Rev. B 86, 060408 (2012).

[7] A. Mitra, Phys. Rev. B 87, 205109 (2013).

[8] M. van den Worm, B. Sawyer, J. Bollinger, and M. Kastner, New J. Phys. 15, 083007 (2013).

[9] J. Marino and A. Silva, Phys. Rev. B 89, 024303 (2014).

[10] F. H. L. Essler, S. Kehrein, S. R. Manmana, and N. J. Robinson, Phys. Rev. B 89, 165104 (2014).

[11] J. Berges, AIP Conf. Proc. 739, 3 (2004).

[12] A. Polkovnikov, K. Sengupta, A. Silva, and M. Vengalattore, Rev. Mod. Phys. 83, 863 (2011).
[13] M. A. Cazalilla, R. Citro, T. Giamarchi, E. Orignac, and M. Rigol, Rev. Mod. Phys. 83, 1405 (2011).

[14] T. Kinoshita, T. Wenger, and D. S. Weiss, Nature (London) 440, 900 (2006).

[15] R. Barnett, A. Polkovnikov, and M. Vengalattore, Phys. Rev. A 84, 023606 (2011).

[16] T. Kitagawa, A. Imambekov, J. Schmiedmayer, and E. Demler, New J. Phys. 13, 073018 (2011).

[17] M. Gring, M. Kuhnert, T. Langen, T. Kitagawa, B. Rauer, M. Schreitl, I. Mazets, D. A. Smith, E. Demler, and J. Schmiedmayer, Science 337, 1318 (2012).

[18] M. Stark and M. Kollar, arXiv:1308.1610.

[19] M. Kollar, F. A. Wolf, and M. Eckstein, Phys. Rev. B 84, 054304 (2011).

[20] M. Rigol, V. Dunjko, V. Yurovsky, and M. Olshanii, Phys. Rev. Lett. 98, 050405 (2007).

[21] M. A. Cazalilla, Phys. Rev. Lett. 97, 156403 (2006).

[22] A. Iucci and M. A. Cazalilla, Phys. Rev. A 80, 063619 (2009).

[23] N. Nessi and A. Iucci, Phys. Rev. B 87, 085137 (2013).

[24] M. A. Cazalilla, A. Iucci, and M. C. Chung, Phys. Rev. E 85, 011133 (2012).

[25] S. A. Hamerla and G. S. Uhrig, Phys. Rev. B 89, 104301 (2014).

[26] M. Rigol, V. Dunjko, and M. Olshanii, Nature (London) 452, 854 (2008).

[27] A. Houghton and J. B. Marston, Phys. Rev. B 48, 7790 (1993).

[28] A. Houghton, H. J. Kwon, and J. B. Marston, Phys. Rev. B 50, 1351 (1994).

[29] A. Houghton, H. J. Kwon, and J. B. Marston, Adv. Phys. 49, 141 (2000).

[30] A. H. Castro Neto and E. H. Fradkin, Phys. Rev. Lett. 72, 1393 (1994).

[31] A. H. Castro Neto and E. H. Fradkin, Phys. Rev. B 49, 10 877 (1994).

[32] A. H. Castro Neto and E. H. Fradkin, Phys. Rev. B 51, 4084 (1995).

[33] P. Kopietz, Bosonization of Interacting Fermions in Arbitary Dimensions (Springer, New York, 1997).

[34] M. Lu, N. Q. Burdick, and B. L. Lev, Phys. Rev. Lett. 108, 215301 (2012).

[35] K. Aikawa, A. Frisch, M. Mark, S. Baier, R. Grimm, and F. Ferlaino, Phys. Rev. Lett. 112, 010404 (2014).

[36] See Supplemental Material at http://link.aps.org/ supplemental/10.1103/PhysRevLett.113.210402 for details of the calculations of the Fermi-surface bosonization and perturbative approaches.

[37] G. Baym and C. Pethick, Landau Fermi-Liquid Theory (Wiley, New York, 1991); S. A. Hamerla and G. S. Uhrig, New J. Phys. 15, 073012 (2013).

[38] T. Barthel and U. Schollwöck, Phys. Rev. Lett. 100, 100601 (2008).

[39] C. Karrasch, J. Rentrop, D. Schuricht, and V. Meden, Phys. Rev. Lett. 109, 126406 (2012); J. Rentrop, D. Schuricht, and V. Meden, New J. Phys. 14, 075001 (2012); F. Pollmann, M. Haque, and B. Dóra, Phys. Rev. B 87, 041109 (2013); D. M. Kennes and V. Meden, Phys. Rev. B 88, 165131 (2013).

[40] A. Mitra and T. Giamarchi, Phys. Rev. Lett. 107, 150602 (2011).

[41] P. Calabrese and J. Cardy, Phys. Rev. Lett. 96, 136801 (2006).

[42] G. Carleo, F. Becca, L. Sanchez-Palencia, S. Sorella, and M. Fabrizio, Phys. Rev. A 89, 031602 (2014). 